\title{
Universal linear motor driven Leg Press Dynamometer and concept of Serial Stretch Loading
}

\author{
Dušan Hamar \\ Faculty of Physical Education and Sports, Comenius University, Bratislava, Slovakia
}

\begin{abstract}
Paper deals with backgrounds and principles of universal linear motor driven leg press dynamometer and concept of serial stretch loading. The device is based on two computer controlled linear motors mounted to the horizontal rails. As the motors can keep either constant resistance force in selected position or velocity in both directions, the system allows simulation of any mode of muscle contraction. In addition, it also can generate defined serial stretch stimuli in a form of repeated force peaks. This is achieved by short segments of reversed velocity (in concentric phase) or acceleration (in eccentric phase). Such stimuli, generated at the rate of $10 \mathrm{~Hz}$, have proven to be a more efficient means for the improvement of rate of the force development. This capability not only affects performance in many sports, but also plays a substantial role in prevention of falls and their consequences. Universal linear motor driven and computer controlled dynamometer with its unique feature to generate serial stretch stimuli seems to be an efficient and useful tool for enhancing strength training effects on neuromuscular function not only in athletes, but as well as in senior population and rehabilitation patients.
\end{abstract}

Key Words: leg press dynamometer, linear motor, serial stretch loading, resistance trainng Eur J Transl Myol - Basic Appl Myol 2015; 25 (4): 215-219

I spent a great deal of my professional life under communist regime. At that time we had very limited access to western technology. That "inspired" us to build up our own diagnostic and training devices, which have been used for the functional assessment of athletes as well as patients. After the "velvet revolution" in 1989, our colleagues from abroad started to visit our institution. Seeing our "do-it-yourself" equipments and possibilities of their application, they expressed wishes to use some of them in their labs. Such recognition motivated us to continue our innovation activities even more intensively. ${ }^{1-3}$

In previous 30 years we have accomplished more than dozen projects, for example isokinetic cycle ergometer, which monitors crank forces. One of these has been bought by Coca Cola Company for Hermann Mayer to be used during the rehabilitation after his motorcycle injury. Many of them are used not only in Slovakia, but also in Germany and Austria. It has even found its way to Japan. Some further examples of isokinetic devices are crank, wheel chair, swim, paddle and rowing ergometers. We have also built treadmill, which monitors the ground reaction forces. Another system called "Drag force and power check", measures the drag force and power while running on the motor driven treadmill. We have even modified the system to register drag force and power while skating on a broad treadmill with artificial ice belt (skatemill). For the application in the practice of weight training we have developed an isoinertial dynamometer, a system able to precisely monitor force, velocity, position and power while lifting the barbell of a known mass.

Within the INTEREG project we have developed three systems. Two for the assessment of balance (a dynamic and task oriented posturography) and the thir for the assessment and training of strength. ${ }^{4-6}$

\section{Universal linear motor driven leg press dynamometer}

Strength and power are not only critical factors affecting sport performance, but are nowadays considered to be important also for the general population, mainly the elderly. This is one of the reasons why, after dealing mostly with cardiorespiratory physiology in early years of my professional carrier, I have directed my interests to strength and power. In a background of the system was the hypothesis that eliciting repeated force peaks during muscle contractions, by means of special dynamometer, may enhance resistance training outcome. The first system we have built for this 


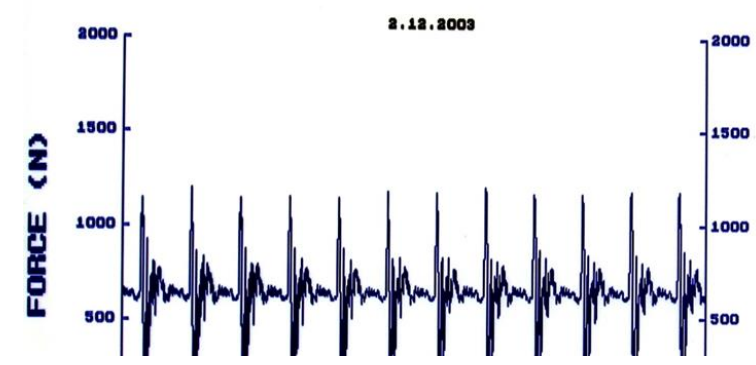

Fig 1. Ground reaction force while standing on the dynamometric platform under influence of counter shock produced at the rate of $10 \mathrm{~Hz}$.

purpose was based on wheel with eccentric "lump". Rotation of this wheel under force platform produced "counter shocks". While standing on it, instead of a straight line corresponding to gravitational, there are periodically repeating force peaks (Fig.1). These additional stimuli are reflected by higher IEMG activities indicating larger simultaneous activation of muscles involved. In one study we were comparing classic lifting training (squats) with the one carried out in the same ways, however on the platform with additional "serial stretch stimuli" produced by a system as described above. Curve of ground reaction forces produced during two squats performed with additional stimuli shows peaks reaching almost double values as compared with squats on a stable platform (Fig. 2).

After an eight-week intervention both groups improved their maximum isometric force without any significant difference between them. However, there was a significant difference in the improvement of the capability to produce force in initial $200 \mathrm{~ms}$ of maximal voluntary contraction. Additional force peaks were likely responsible for a more pronounced training effect on the rate of force development. Similar responses to training using the above mentioned device have been shown also in untrained elderly subjects.

Device used for these studies is rather simple and relatively cheap to build, however, if we wanted to manipulate force peaks (to increase the intensity of stimulation) we have to use faster rotation. This logically changes also the frequency of stimulation. Save complicated mechanical modification, this system does not allow to control independently amplitude and frequency of mechanical stimuli. That is why we were looking for some more sophisticated alternative.

In fact it was my dream for years to build a diagnostic and training device featuring the possibility of separate control of all biomechanical variables involved in strength exercise. However, because of financial constraints I was not able to accomplish such a project. After several years of cooperation with Professor $\mathrm{H}$.

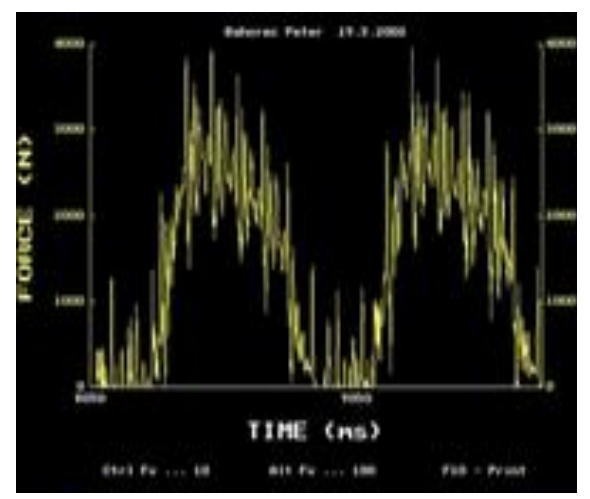

Fig 2. Ground reaction force while performing two squats with and without counter shocks produced at the rate of $10 \mathrm{~Hz}$.

Kern I told him about my dream. I remember the exact words of his reply: "Wir machen das, koste es, was es wolle" (We build it, no matter what the cost will be"). So I had got free hands and we built a universal linear motor based leg press dynamometer (Fig. 3). Some experts on the field, after seeing the machine and hearing the amount of money we spent to build it, made a remark that "should a serious company like Siemens be contracted for such a project, that amount would not be enough to cover initial projection phase". So, what are the features of the machine? The crucial parts are two computer controlled linear motors mounted to the horizontal rails. Motors selected can accelerate at $15 \mathrm{~g}$ (almost $150 \mathrm{~m} / \mathrm{s}^{2}$ ) and can be controlled in such a way that they keep constant resistance force in selected position or velocity in both directions. Because of high breaking capacity (up to 2 times $1800 \mathrm{~N}$ ) they can keep constant velocity regardless of force applied in both, concentric or eccentric phase of muscle contraction. Linear motors have been complemented with two pedals, each equipped with special strain gauge transducers in order to register force generated by muscles. System also incorporates precise linear encoder for the registration of pedals position. After an appropriate processing, encoder signal also provides velocity (integration) and acceleration (double integration). Multiplying force by velocity yields the value of instant power. In this way the system captures all basic biomechanical parameters involved in resistance exercise.

Why is the system universal? It is because of its ability to simulate any mode of muscle contraction. If we "tell" the system to keep constant resistance, the pedals stay in that position, until the subjects' muscles exceed the force set. From that moment on concentric movement starts and lasts until force exerted by muscles does not drop below preset value. From that moment a reversed movement starts and lasts until either initial position is reached or force produced by muscles again exceeds the pre set value. This constant 


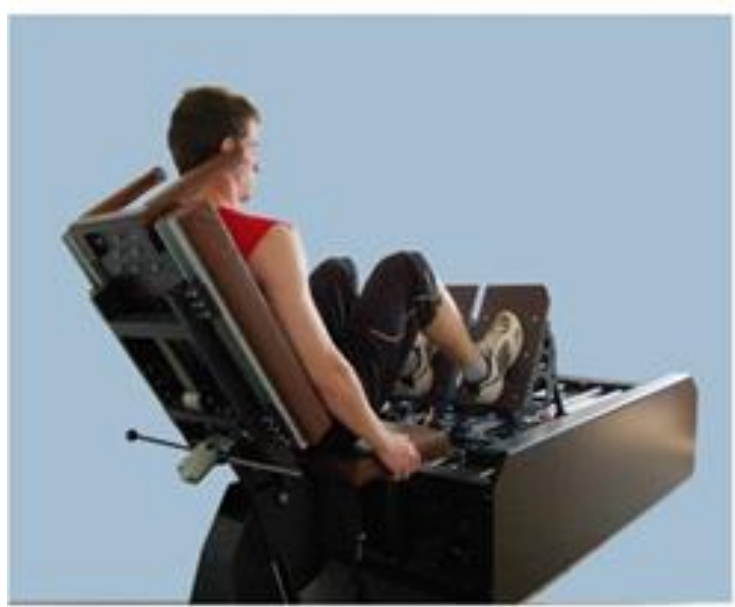

Fig 3. Universal computer controlled linear motor based leg press dynamometer.

resistance mode actually simulates the situation while lifting the weights.

If the maximal breaking force is set in constant resistance mode, so that a subject is not able to move the pedals, the system actually works in isometric mode. Parameters of isometric contraction can be measured in any preset position.

Constant velocity, or isokinetic mode, can easily be implemented by setting the velocity separately for concentric and eccentric phase of muscle contraction. The system accommodates the braking force in such a way that velocity of the movement in both concentric and eccentric phases remains constant regardless of force applied to the pedals.

\section{Concept of serial stretch stimulation}

The above mentioned modes of muscle work are not novel. Constant resistance, isometric or isokinetic resistance exercises can be performed on many commercially available dynamometric systems. Uniqueness of our leg press machine lies in the capability to precisely generate defined serial stretch stimuli in a form of repeated force peaks during ordinary isokinetic contraction.

How do we achieve such peaks? One of the basic principles of muscle physiology says that force produced by the muscle decreases with velocity of contraction (Fig. 4). If one wants to increase the force generated by concentric contraction, he has to suddenly decrease, stop or reverse the velocity of pedals. So, if one is extending the legs while performing concentric contraction and pedals suddenly move back just a small distance, e.g. $0.5 \mathrm{~cm}$, his or her muscles will generate a force peak.

The situation is different during eccentric contraction. Decrease, stop or even reverse of velocity would take a part of resistance force away and there would be no peaks, but decrease of the force generated by the

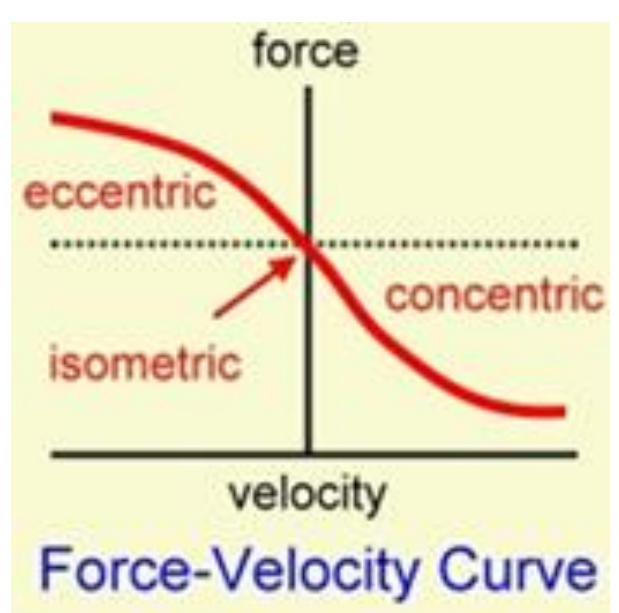

Fig 4. Force generated by muscles at different velocity of contraction.

muscles. To elicit peaks, one has to impose a short period of accelerated eccentric movement. In fact, in absolute values this means decreasing the velocity of contraction, what is in line with the principle of muscle physiology describing a relationship between force and velocity during maximal muscle contraction (Fig 4).

Figure 5 shows one leg press repetition performed with maximal effort in classical isokinetic mode and in a mode producing serial stretch stimuli. Subject starts the movement from knee flexion and after extending the lower extremities (concentric phase) returns back to initial position (eccentric phase). As one can see, force produced in eccentric phase is higher than the one in concentric one. If the additional counter movements in concentric phase and small acceleration phases in eccentric phase are implemented, peaks, substantially exceeding force produced during isokinetic contraction, occur.

What is the advantage of such a system? One can control force peaks (and amount of additional stimulation) by changing the velocity of counter movement in concentric and speed up the segments in the eccentric phase. The bigger the change of velocity, the higher the force peaks. It is also possible to modulate how fast the system changes the velocity. Faster change means higher peaks and vice versa. By manipulating these variables one can control the force peaks in quite a large range. They can be very low or extremely high and evoked in various frequencies.

At the beginning we didn't know what frequency and magnitude of force peaks to apply in order to achieve the best training results in athletes or patients. In the first study we used setup producing the peaks about $100 \%$ above the force generated during classical isokinetic contraction applied at the rate of $10 \mathrm{~Hz}$. The study has been carried out on athletes as they had no problems to cope with this kind of additional stimulation. Two groups of athletes performed similar 


\section{Leg Press Dynamometer and concept of Serial Stretch Loading}

Eur J Transl Myol - Basic Appl Myol 2015; 25 (4): 215-219

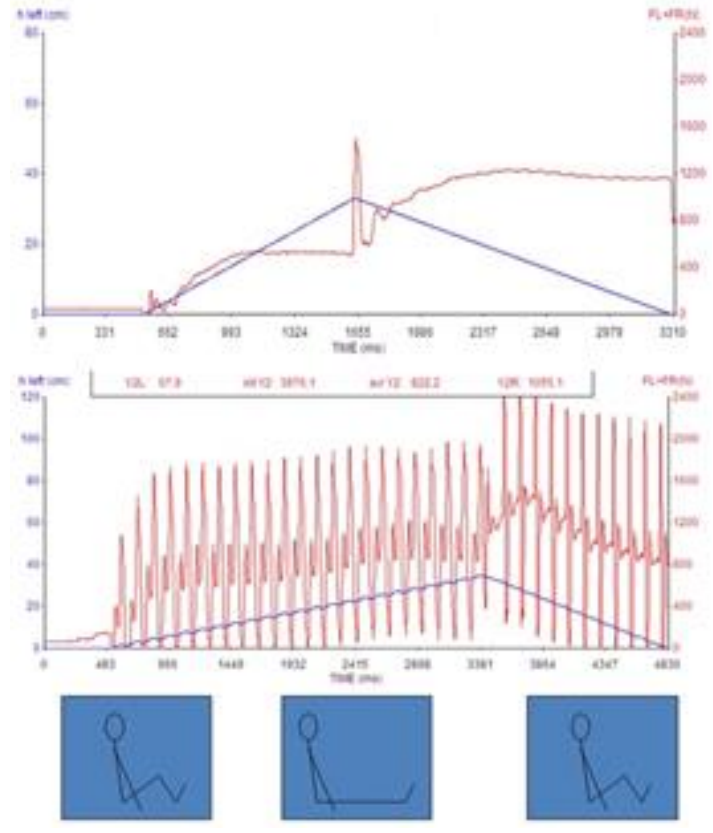

Fig 5. Force and pedal position during one leg press repetition in classical isokinetic and serial stretch loading mode.

8-week resistance training programs, one in isokinetic mode the other with additional serial stretch stimuli.

What were the main outcomes? There was no significant difference in the increase of the maximum isometric force indicating that both training modalities posses the capability to increase maximum force. However, there was a substantial difference in the rate of force development. This means that additional serial stretch stimuli enhance the training effect on the capability to produce force at the very beginning of the muscle contraction. Using modified setup eliciting lower force peaks for elderly and patients, e.g. after hip replacement, follow up studies showed similar efficiency as in athletes.

What is a practical meaning of improving the rate of force development? As in many sports an athlete has a limited time (usually less than $200 \mathrm{~ms}$ ) to apply the force, in terms of performance, the rate of force development is more relevant than maximal isometric force itself. The capability to generate high force in initial phase of muscle contraction plays also an important role in injury prevention. External forces acting on the body structures, e.g. twisting ankle, can be compensated not only by passive tissues like joint capsule and ligaments but also by an action of the muscles controlling the movement of that particular joint. If the muscle contraction is fast enough, it can help to protect structures under strain. However, should muscle contraction take too long, the protecting effect would not apply and injury is more likely to happen.
Furthermore, in sports, as well as in daily life, a correction of disturbed balance is often needed. Efficient correction also requires fast muscle contraction. Slowly contracting muscles do not provide an efficient compensation of disturbed balance to prevent falls and resulting injuries. This is namely a problem of elderly population, in which falls and associated complications are so frequent that they pose not only medical, but a socio-economical problem.

\section{Conclusions}

In conclusion, a universal linear motor driven and computer controlled dynamometer with its unique feature to generate serial stretch stimuli seems to be an efficient and useful tool for enhancing strength training effects on neuromuscular function not only in athletes, but as well as in senior population and rehabilitation patients.

\section{Acknowledgement}

This work was supported by European Regional Development Fund - Cross Border Cooperation Programme Slovakia - Austria 2007-2013 (InterregIVa), project Mobilität im Alter, MOBIL, N_00033 (partners: Ludwig Boltzmann Institute of Electrical Stimulation and Physical Rehabilitation, Austria, Center for Medical Physics and Biomedical Engineering, Medical University of Vienna, Austria, and Faculty of Physical Education and Sports, Comenius University in Bratislava, Slovakia); Austrian national co-financing of the Austrian Federal Ministry of Science and Research; Ludwig Boltzmann Society (Vienna, Austria).

This paper is a transcription of the talk presented to the Interreg IVa Final Meeting November 8 2014, Vienna, Austria.

\section{Corresponding Author}

Dušan Hamar, Faculty of Physical Education and Sports, Comenius University, Bratislava, Slovakia

E-mail: dusan.hamar@uniba.sk

\section{References}

1. Hamar D, Zemkova E, Böhmerova L. Effect of propriceptive stimulation on motor capabilities in elderly women, Medicina Sportiva Bohemica et Slovaca 2005;14:166-74.

2. Willmore JH, Costill DL. Physiology of Sport and Exercise, 1994, Human Kinetics, Champain, 549 pp 47-47.

3. Cvecka J, Hamar D, Trimmel L, Vogelauer M, Bily W, Löfler S, Drewniak P, Grim-Stieger M, Kern H: The influence of serial stretch loading on the effectiveness of isokinetic strength training. Basic Appl Myol 2009;19:175-18.

4. Mosole S, Carraro U, Kern H, Loefler S, Fruhmann H, Vogelauer M, Burggraf S, Mayr W, Krenn M, Paternostro-Sluga T, Hamar D, 


\section{Leg Press Dynamometer and concept of Serial Stretch Loading}

Eur J Transl Myol - Basic Appl Myol 2015; 25 (4): 215-219

Cvecka J, Sedliak M, Tirpakova V, Sarabon N, Musarò A, Sandri M, Protasi F, Nori A, Pond A, Zampieri S. Long-term high-level exercise promotes muscle reinnervation with age. J Neuropathol Exp Neurol. 2014;73:284-94. doi: 10.1097/NEN.0000000000000032.

5. Zemková E, Jeleň M, Kováčiková Z, et al. Power outputs in the concentric phase of resistance exercises performed in the interval mode on stable and unstable surfaces. J Strength Cond Res. 2012;26:3230-6. doi: 10.1519/JSC.0b013e31824bc197.

6. Kern H, Pelosi L, Coletto L, et al. Atrophy/hypertrophy cell signaling in muscles of young athletes trained with vibrationalproprioceptive stimulation. Neurol Res. 2011;33:998-1009.

doi: 